\title{
Cannabinoid Receptor 2 Expression in Human Proximal Tubule Cells is Regulated by Albumin Independent of ERK1/2 Signaling
}

\author{
Kayte A. Jenkin ${ }^{a}$ Andrew J McAinch ${ }^{\mathrm{a}}$ Jessica F. Briffa ${ }^{\mathrm{a}, \mathrm{b}} \quad$ Yuan Zhang ${ }^{\mathrm{c}}$ \\ Darren J. Kellyc Carol A. Pollock ${ }^{d}$ Philip Poronnik ${ }^{\mathrm{e}}$ Deanne H. Hryciwa,
}

aBiomedical and Lifestyle Diseases Unit, Victoria University, College of Biomedical and Health Sciences; ${ }^{\mathrm{b}}$ Department of Physiology, The University of Melbourne, Melbourne; 'Department of Medicine, St Vincent's Hospital, Fitzroy, VIC; 'Kolling Institute of Medical Research, Sydney Medical School, University of Sydney, Royal North Shore Hospital, St Leonards; eSchool of Medical Sciences, The Bosch Institute, The University of Sydney, NSW, Australia

\section{Key Words}

Proximal tubule $•$ Cannabinoid receptor CB2 $・$ Diabetic nephropathy

\begin{abstract}
Background: The cannabinoid receptor type 2 (CB2) is reduced in podocytes of animals and humans with Type 2 Diabetes Mellitus (T2DM), with activation of CB2 ameliorating albuminuria in animals. As albuminuria also is due to proximal tubule dysfunction, the aim of this study is to investigate tubular expression of CB2 under diabetic conditions in addition to the cell signaling pathways that underlie these changes. Methods: We characterized total CB2 protein in diabetic animals and in Human Kidney 2 (HK2) cells exposed to elevated albumin and glucose, the levels of CB2 mRNA and protein. We also used latrunculin to determine if internalization of albumin was required to regulate CB2 levels. Finally, we characterized the levels of active and total AKT, ERK1/2 and p38 in response to albumin. Results: There were no changes to CB2 expression in kidney lysate from diabetic rats. In HK2 cells, expression of CB2 was unaltered following exposure to high glucose. High albumin treatment alone and in combination with high glucose, resulted in a significant reduction in CB2 receptor mRNA expression at 6 and 18 hours. CB2 protein expression was reduced at 6 and 24 hours, in high albumin and in combination with high glucose. Internalization of albumin was required to regulate CB2 levels, and inhibition of ERK1/2, did not rescue the loss of CB2 in response to albumin. Conclusion: We have demonstrated that internalization of albumin is required to reduce $C B 2$ mRNA and protein expression in proximal tubules in vitro. Consequently, altered expression of $\mathrm{CB} 2$ in both the podocytes and tubules may contribute to the albuminuria observed in T2DM.




\section{Cellular Physiology Cell Physiol Biochem 2013;32:1309-1319 \begin{tabular}{l|l} 
and Biochemistry Published online: November 22, 2013 & $\begin{array}{l}\text { C } 2013 \text { S. Karger AG, Basel } \\
\text { www.karger.com/cpb }\end{array}$ \\
\hline
\end{tabular} Jenkin et al.: Albumin Modulates CB2 in Proximal Tubule Cells}

\section{Introduction}

Hyperglycemia is an etiologic factor for the development of diabetic nephropathy [1, 2]. On the cellular level, hyperglycemia leads to the accumulation of extracellular matrix, thickening of the glomerular and tubular basement membrane, glomerulosclerosis and tubular fibrosis which leads to hyperfiltration through the glomerulus [1]. Hyperglycemia activates a number of key inflammatory cytokines which are produced by cells of the kidney during renal damage [3], which results in elevated levels of protein, specifically albumin, in the filtrate [1]. Microalbuminuria is the earliest clinical indicator of diabetic nephropathy, and it is associated with an increased risk for the development of kidney failure [4]. Diabetic nephropathy increases the risk of developing end stage renal failure [1], with a number of molecular targets identified as playing a role in diabetic pathophysiology. Recent studies focused on the endocannabinoid system [5] and its role in diabetic nephropathy $[2,6-8]$. The endocannabinoid system is comprised of the endogenous lipid transmitters anandamide (AEA) and 2-arachidonoyl glycerol (2-AG) which act upon a select number of receptors including the cannabinoid receptors 1 and 2 (CB1 and CB2) [9]. This system is involved in energy homeostasis, glucose and lipid metabolism, energy intake and expenditure [10]. Research has demonstrated that modulation of the endocannabinoid system can reduce damage associated with diabetic nephropathy $[2,6,8,11,12]$.

Recently, a study has focused on one specific member of the cannabinoid family, namely CB2 and its role in diabetic nephropathy. The CB2 receptor is expressed in both rat and human kidneys [2], however it is more abundant in immune cells and splenic tissue [13]. CB2 expression is altered in diabetic nephropathy. In humans with diabetic nephropathy, CB2 expression is reduced in glomerular podocytes and the endocannabinoid agonist 2-AG is reduced in the kidney [2]. Further, in a diabetic animal model, CB2 expression is reduced in podocytes [2]. However interestingly, the level of CB2 mRNA in diabetic whole kidneys is unaltered compared to control [2], perhaps due to recruitment of inflammatory cells in renal tissue. However, expression of CB2 specifically in proximal tubule cells exposed to hyperglycemic conditions is unknown.

Altered expression of CB2 mRNA and protein in podocytes is important in the regulation of the normal physiological processes in the glomerulus. Specifically, a study has shown that in an animal model of diabetes, treatment with CB2 agonist, AM1241, reduced podocyte protein loss and ameliorated albuminuria [2]. Further, treatment with AM1241 ameliorated albuminuria in a diabetes model. Thus, these in vivo data has demonstrated a protective role for the CB2 receptor signaling in albuminuria associated with diabetic nephropathy [2]. It is important to note that albuminuria is the result of both glomerular and proximal tubule dysfunction in vivo [14]. The link between activation of the receptor and altered albumin handling by the glomerulus has clearly been established; however the contribution of CB2 to tubular processing of albumin has not received much attention.

The serine/threonine kinase AKT protein has been identified as an important regulator of renal hypertrophy and apoptosis induced by hyperglycemia [15]. Downstream, phosphorylation of AKT leads to the activation of a signaling cascade which regulates cell proliferation, migration, differentiation and cell survival, known as the mitogen activated protein kinase (MAPK) pathway. The MAPK cascade subsumes several proteins, including p38 and ERK1/2 which have been demonstrated to be involved in the pathogenesis of diabetic nephropathy [15-17]. Activity of the CB2 receptor has been linked to the AKT/MAPK signaling pathway $[18,19]$. In vitro CB2 activation results in reduced phosphorylated ERK1/2 protein expression [19] and conversely, CB2 antagonists can lead to greater and sustained activation of MAPK proteins [18], indicating that the mechanism of the CB2 receptor in diabetic nephropathy may mediate changes via the AKT/MAPK signaling pathway. The association between CB2 expression and AKT/MAPK has not been examined in the role of renal tubular damage in diabetic nephropathy.

In the early stages of diabetic nephropathy, downstream renal proximal tubule cells are exposed to high levels of glucose, with increases in hyperfiltration leading to elevated 
albumin and glucose in the filtrate and altered AKT/MAPK signaling [17, 20]. Therefore, the aims of this study were to determine: 1 . expression of the CB2 receptor and its relationship with AKT/MAPK signaling in proximal tubule cells mimicking the diabetic phenotype, 2. determine if CB2 expression alters tubular processing of albumin and if so, 3. characterize whether CB2 receptor expression in proximal tubule cells is dependent on albumin internalization.

\section{Materials and Methods}

\section{Diabetic kidney}

Six-week old male Sprague-Dawley (SD) rats were randomized to receive either $55 \mathrm{mg} / \mathrm{kg}$ of streptozotocin (STZ; Sigma, USA) diluted in $0.1 \mathrm{M}$ citrate buffer $\mathrm{pH} 4.5$ or citrate buffer (control) by tail vein injection following an overnight fast. 16 weeks after STZ treatment animals were killed and kidney tissues harvested. Experimental procedures adhered to the guidelines of the National Health and Medical Research Council of Australia's Code for the Care and Use of Animals for Scientific Purposes and were approved by the Animal Research Ethics Committee of St. Vincent's Hospital.

\section{Cell Culture}

Human proximal tubule cell line (HK2) and opossum kidney ( $\mathrm{OK}$ ) cells were maintained as previously described [21]. Cells were grown to $80 \%$ confluence incubated in $5 \mathrm{mM}$ glucose DMEM/F12 media without FBS (control) for 24 hours. Cells were then treated for 4, 6, 18 and 24 hours with control media or high glucose $(25 \mathrm{mM})$, high albumin media $(1 \mathrm{mg} / \mathrm{ml})$ or both high glucose $(25 \mathrm{mM})$ and high albumin $(1 \mathrm{mg} / \mathrm{ml})$. These concentrations have previously been published in proximal tubule cells lines and were selected to mimic the concentration of glucose and albumin that the proximal tubule would be exposed to in a diabetic and proteinuric states [22].

\section{Real time PCR}

In HK2 cells, following treatment, mRNA was extracted and real time PCR performed using previously described methods [21]. CB2 (Accession number NM _001841.2) was amplified with forward (5'-3' ACTCCATGGTCAACCCTGTC) and reverse (5'-3' GATCTCGGGGCTTCTTCTTT) primers. Control housekeeping gene, glyceraldehydes-3-phosphate dehydrogenase (GAPDH; accession number NM 002046.3) was also measured using forward (5'-3' CAACGACCACTTTGTCAAGC) and reverse (5'-3' TTACTCCTTGGAGGCCATGT) primers.

\section{Western blot}

HK2 cells were lysed and analysed via Western blot using previously described methods [21]. In addition, kidney lysate from STZ rats was prepared homogenization in HNT buffer (containing $20 \mathrm{mM}$ HEPES, $120 \mathrm{mM} \mathrm{NaCl}, 0.6 \%$ Triton-X, 5 mM EDTA and protease inhibitor cocktail).

$40 \mu \mathrm{g}$ of protein from HK2 lysate and $100 \mu \mathrm{g}$ of whole kidney lysate was analysed via Western blot as described previously [21]. A polyclonal antibody for CB2 was purchased from Cayman Chemicals (Ann Arbour, MI), antibodies for phospho AKT, AKT, phosphor p38, p38, phosphor ERK1/2 and ERK1/2 were purchased from Cell Signalling Technology (Beverly, MA). Secondary antibodies anti-mouse and anti-rabbit were purchased from Sigma Aldrich (St Loius, MO). Primary antibodies were used at 1:1000 dilution and secondary antibodies were diluted 1:2000. CB2 protein expression was relative to $\beta$-Actin (Sigma Aldrich, St Louis, MO), and expressed as \% of control. For analysis of phosphorylated protein, the ratio of phosphorylated to total protein was determined.

\section{Albumin Uptake}

To measure the effect of CB2 and MAPK regulation of albumin uptake, opossum kidney cells were exposed to albumin conjugated to Texas Red $(50 \mu \mathrm{g} / \mathrm{ml})$ in the presence of specific inhibitors for two hours at $37^{\circ} \mathrm{C}$ as previously described [23] CB2 inhibitor AM630 (Cayman Chemical, Ann Arnour, MI) was used at 10 $\mu \mathrm{M}$ concentration. ERK1/2 inhibitor U0126 (10 mM) and p38 inhibitor SB203580 (25 $\mu \mathrm{M})$ were purchased from Cell Signalling Technology (Beverly, MA). Inhibitors were used at concentrations recommended by 


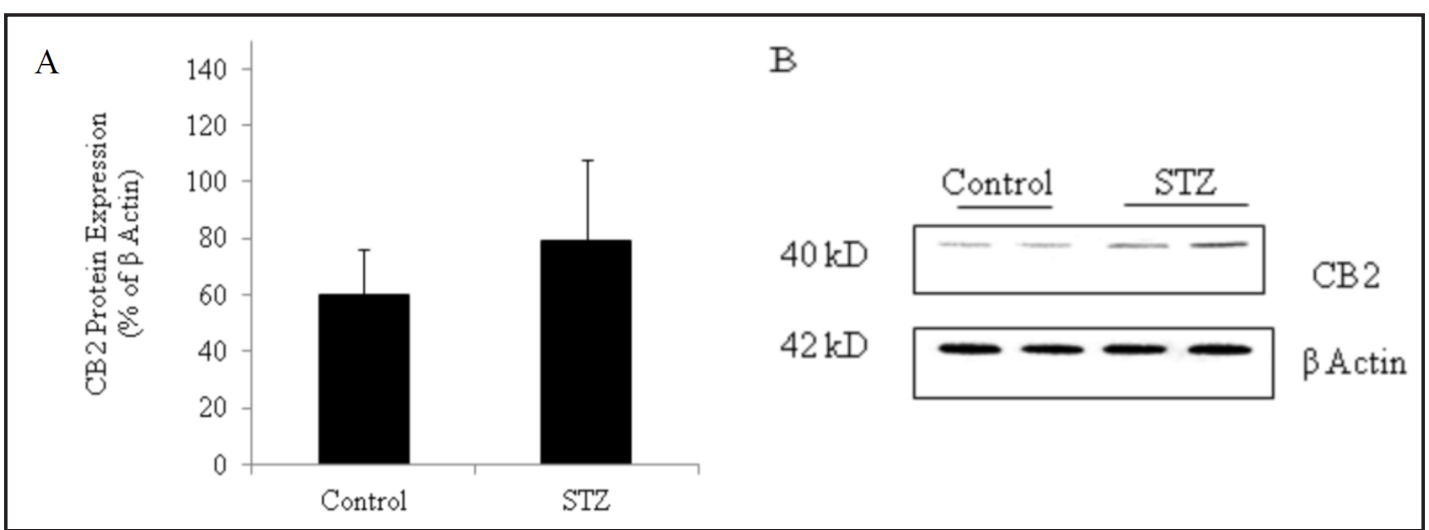

Fig. 1. A. Densitometric quantification of the CB2 receptor expression in whole kidney lysate from control and diabetic STZ treated animals (data expressed as mean \pm SEM) B. Representative Western Blot of CB2 receptor expression in Control and STZ kidney lysate with loading control $\beta$-Actin. $\mathrm{N}=8$.

manufacturer's instructions. At the end of the albumin uptake period, cells were washed in HEPES buffer and lysed in MOPS buffer (20 mM MOPS, $0.1 \%$ Triton X-100, pH 7.4). TR-albumin florescence was determined using an $\mathrm{xMark}^{\mathrm{TM}}$ Microplate Absorbance Spectrophotometer (Bio-Rad) at $580 \mathrm{~nm}$ excitation and $630 \mathrm{~nm}$ emission wavelengths. TR-albumin uptake was adjusted for background and standardized to treatment without the presence of inhibitors (control).

To determine whether the expression of CB2 is dependent on albumin internalisation, HK2 cells were exposed to latrunculin to fragment the cytoskeleton and thus inhibit albumin endocytosis, according to previously described published methods with minor modifications [23]. Briefly, HK2 cells were treated for six hours with $1.5 \mu \mathrm{M}$ latrunculin A (Sigma Aldrich, St Loius, MO) with control, high glucose, high albumin or both high glucose and albumin media, as outlined in the previous section. Cells were then lysed and analysed for CB2 receptor expression with western blot analysis.

\section{Statistical Analysis}

Data is presented as mean \pm SEM. Statistical significance (considered when $\mathrm{p}<0.05$ ) was determined using an independent samples T-Test or One-Way ANOVA, with Tukey's post hoc analysis used to determine differences between groups.

\section{Results}

Protein Expression of CB2 receptor in whole kidney lysate from diabetic rats

Renal expression and function of the CB2 receptor in the diabetic milieu is currently not well understood. Rats rendered diabetic with the injection of STZ had a significant increase in albuminuria, tubulointerstitial fibrosis, peritubular collagen IV accumulation, reactive oxygen species and macrophage infiltration, indicative of diabetic nephropathy [6]. Despite, a previous study demonstrating that podocyte levels of CB2 are reduced in animal and humans with T2DM [2], in whole kidney lysate, there was no difference in CB2 receptor protein levels between STZ and control animals relative to the $\beta$-actin loading control $(n=8$, Fig. 1A and 1B).

mRNA expression of CB2 receptor in proximal tubule cells in vitro

Quantitative 'real time' PCR analysis was used to determine if mRNA of the CB2 receptor is altered in proximal tubule cells exposed to the diabetic milieu. Following 4 and 24 hours exposure to the different treatments, CB2 mRNA expression was unaltered across treatment groups $(n=9)$. 'Real time' PCR analysis showed that proximal tubule cells exposed to high albumin exhibited significantly lower CB2 receptor expression $(\mathrm{p}<0.05)$ following 6 hours $(0.38 \pm 0.12)$ and 18 hours $(0.45 \pm 0.05)$ treatment periods (Fig. 2) compared to control 
Fig. 2. 'Real time' PCR analysis of proximal tubule expression of CB2 receptor in HK2 cells treated with high glucose $(25 \mathrm{mM})$, high albumin $(1 \mathrm{mg} / \mathrm{ml})$ and a combination of high glucose/ high albumin for 4, 6, 18 or 24 hours. GAPDH was used as housekeeping gene. Data expressed as normalized to control treatment (5 mM glucose, no albumin), mean \pm SEM. Significance is indicated by $* \mathrm{p}<0.05$ compared to control treatment. $\mathrm{N}=9$

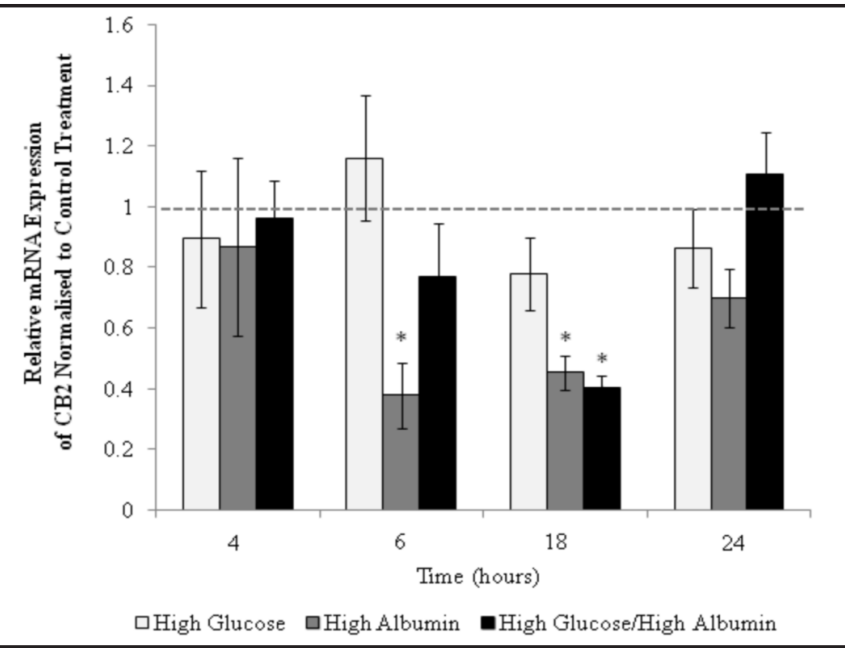

treatment $(1.00 \pm 0.19)$ conditions $(n=9)$. Further, CB2 receptor expression was also significantly reduced $(\mathrm{p}<0.05)$ when high albumin combined with high glucose treatment $(0.40 \pm 0.04)$ was applied for 18 hours $(n=9$, Fig. 2$)$. High glucose treatment alone did not significantly alter CB2 receptor expression when compared to control treatment at any time interval.

Protein Expression of CB2 receptor in proximal tubule cells in vitro

In addition to 'real time' PCR quantification of CB2 mRNA, western blot analysis was used to confirm that alterations protein expression of CB2. At 4 hours (Fig. 3A) and 18 hours (Fig. 3C) there was no significant change in CB2 receptor protein expression. Six hours high albumin treatment alone caused a significant reduction in CB2 receptor protein expression $(0.50 \pm 0.09, p<0.05, n=3$ Fig. $3 B)$ when compared to control treatment. The combination of high glucose and high albumin caused a significant reduction in CB2 protein expression following 6 and 24 hours treatment $0.43 \pm 0.08$ and $0.42 \pm 0.05, p<0.05, n=3$ Fig. 3B and $3 \mathrm{D}$, respectively). High glucose conditions did not significantly alter CB2 expression in HK2 proximal tubule cells at any time interval.

CB2 Receptor expression is regulated by albumin internalization

Proximal tubule cells are exposed to higher levels of albumin in diabetic nephropathy and are responsible for its reabsorption from the filtrate via endocytosis [24]. To determine whether CB2 receptor expression is mediated by exposure to increased albumin alone, or dependent on tubular internalization of albumin, latrunculin $\mathrm{A}(1.5 \mu \mathrm{M})$ was used to disrupt the actin microfilament network and abolish albumin uptake. Here, we demonstrated that when HK2 cells exposed to high glucose and albumin for six hours in conjunction with latrunculin $\mathrm{A}$, there were no significant differences in CB2 receptor expression compared to control ( $n=3$, Fig. 4), indicating that the process of albumin endocytosis by proximal tubule cells is required for CB2 downregulation.

\section{Alterations in AKT/MAPK signaling proteins in proximal tubule cells}

To investigate whether changes in CB2 receptor expression corresponds with changes to AKT/MAPK signaling alterations, HK2 cells treated for six hours with media containing high albumin in the presence and absence of high glucose. Western blot analysis demonstrated that high albumin in the presence and absence of high glucose did not alter AKT phosphorylation, while high albumin alone led to significant increase in phosphorylation of $\mathrm{p} 38(\mathrm{p}<0.05, \mathrm{n}=$ 3, Fig. 5A, 5B and 5C). Phosphorylation of ERK1/2 was significantly increased in HK2 cells treated with both high albumin alone and in combination with high glucose $(p<0.05, n=3$, Fig. $5 \mathrm{~A}$ and $5 \mathrm{D}$ ). These data demonstrate that elevated albumin in the presence and absence of high glucose increases ERK1/2 phosphorylation. 


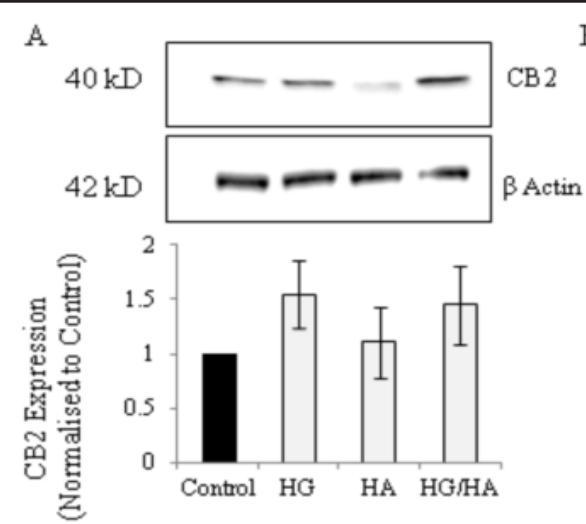

B
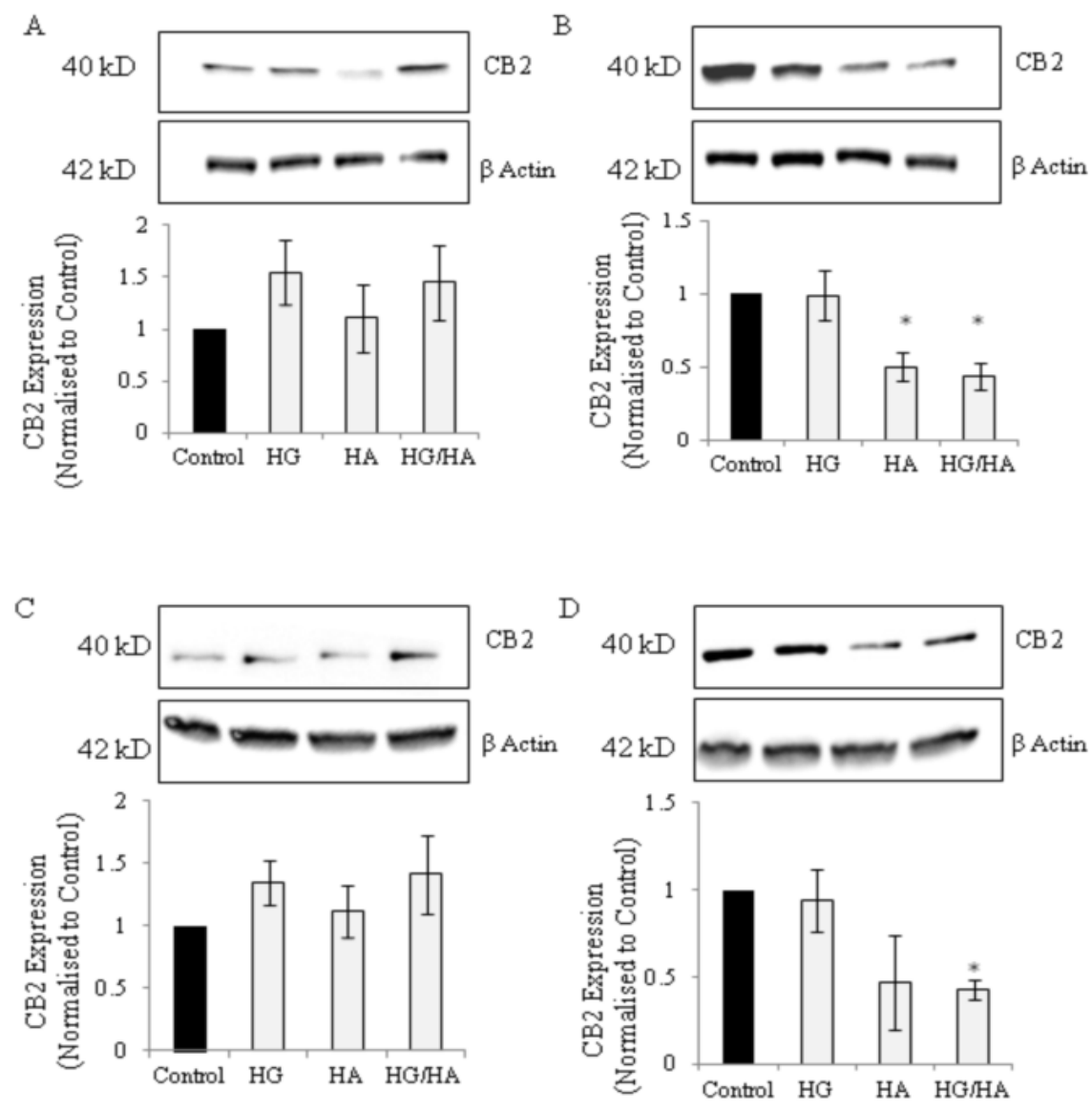

Fig. 3. Representative Western blot and densitometric quantification of CB2 receptor expression in HK2 cells treated with high glucose (HG; $25 \mathrm{mM}$ ), high albumin (HA; $1 \mathrm{mg} / \mathrm{ml}$ ) and a combination of high glucose/ high albumin (HG/HA). Protein abundance (y axis) was normalized to control treatment ( $5 \mathrm{mM}$ glucose, no albumin), data expressed as mean $\pm \mathrm{SEM} . \mathrm{N}=3$. Significance is indicated by $* \mathrm{p}<0.05$ compared to control treatment. A. Treatment for 4 hours B. Treatment for 6 hours. C. Treatment for 18 hours. D. Treatment for 24 hours.

\section{CB2 Receptor expression is not mediated by ERK1/2}

To determine whether CB2 receptor expression is mediated by increased ERK1/2 phosphorylation, HK2 cells were treated for six hours with high glucose and high albumin media in the presence of ERK1/2 inhibitor U0126 $(10 \mu \mathrm{M})$. Treatment with the inhibitor resulted in a significant reduction of $\mathrm{CB} 2$ receptor expression across all treatment groups compared to control ( $p<0.05, n=3$, Fig. 6A). Thus, as inhibition of ERK1/2 does not rescue the reduction in $\mathrm{CB} 2$ protein in response to high albumin, this kinase does not regulate the expression of CB2 in response to albumin.

\section{Regulation of albumin uptake in proximal tubule cells by CB2 and ERK1/2}

We have showed that high albumin leads to increase phosphorylation of ERK1/2 (Fig. 5D) and that endocytosis of albumin in proximal tubule cells is essential for regulating CB2 receptor expression (Fig. 4). To determine whether these cellular alterations lead to changes in proximal tubular handling of albumin, OK cells were treated for 2 hours with TR-albumin in the presence of specific CB2 and ERK1/2 inhibitors. Here, we showed that inhibition of OK cells with CB2 specific antagonist AM630 $(10 \mu \mathrm{M})$ led to a significant increase in albumin uptake of OK cells $(n=8, p<0.05$, Fig. 7A). Inhibition of ERK1/2 with antagonist U0126 (10 $\mu \mathrm{M})$ and MAPK inhibitor SB203580 $(25 \mu \mathrm{M})$ had no significant effect on albumin uptake in 


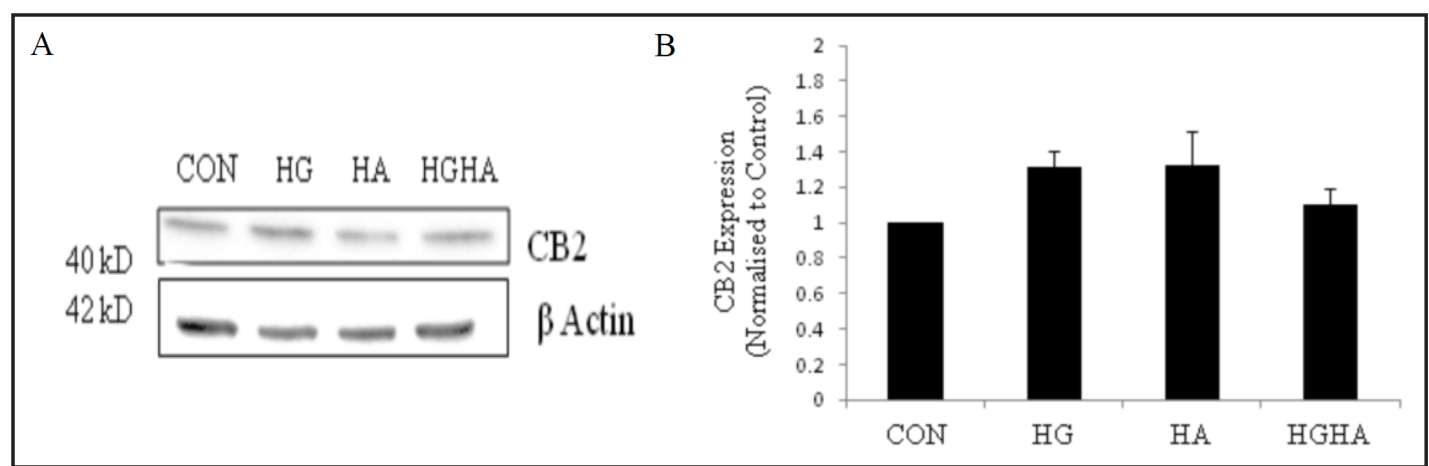

Fig. 4. Representative Western blot and densitometric quantification of CB2 receptor expression in HK2 cells treated with latrunculin A in conjunction with high albumin (HA; $1 \mathrm{mg} / \mathrm{ml}$ ) and a combination of high glucose/ high albumin (HG; $25 \mathrm{mM} / \mathrm{HA}$ ). Protein abundance (y axis) was normalized to control treatment ( $5 \mathrm{mM}$ glucose, no albumin), data expressed as mean \pm SEM. $\mathrm{N}=3$. No significant differences between treatment groups.

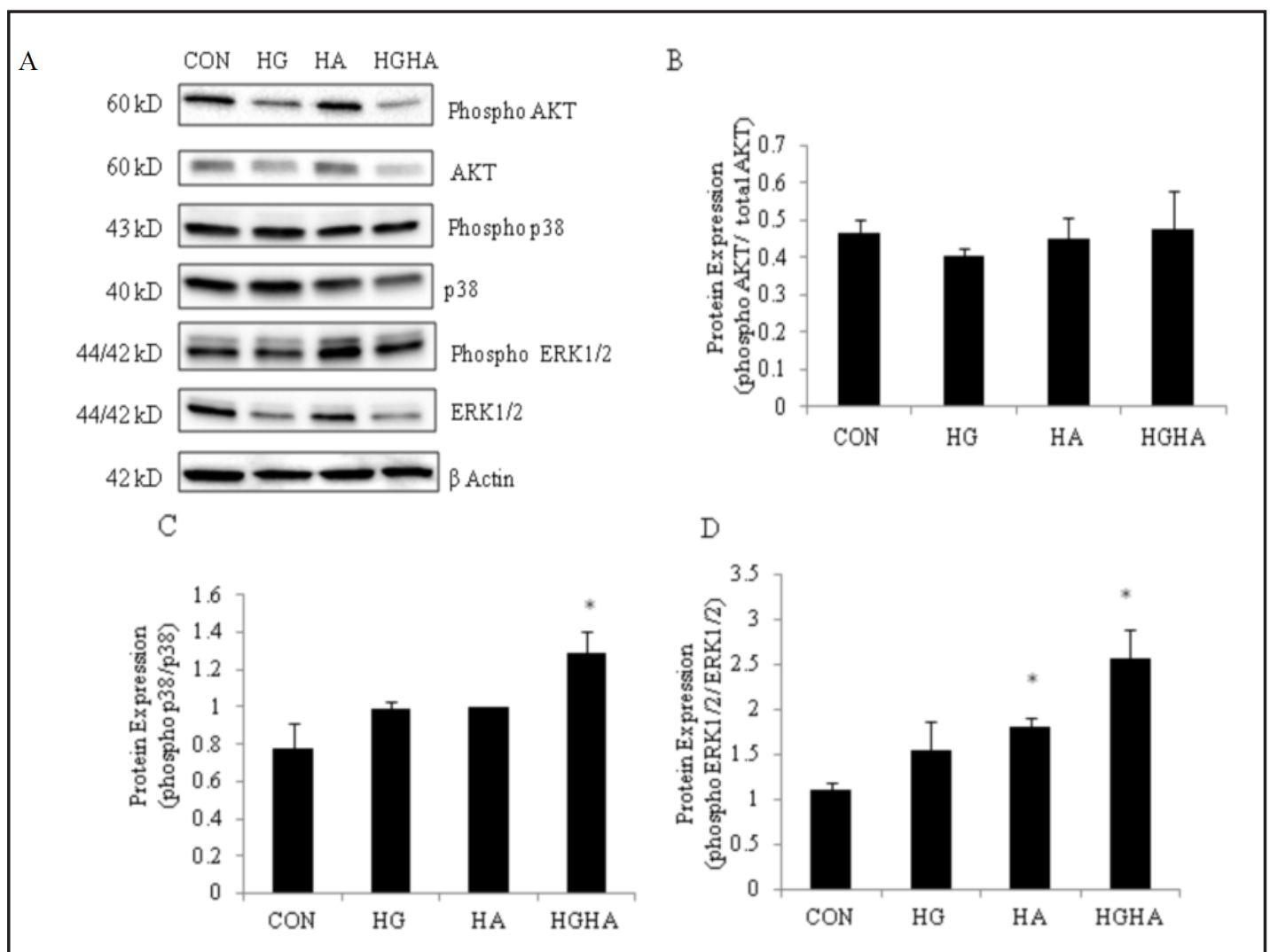

Fig. 5. AKT/MAPK profile in HK2 cells treated with high albumin (HA; $1 \mathrm{mg} / \mathrm{ml}$ ) and a combination of high glucose/ high albumin (HG; $25 \mathrm{mM} / \mathrm{HA}$ ). Protein abundance (y axis) was normalized to control treatment ( $5 \mathrm{mM}$ glucose, no albumin), data expressed as mean \pm SEM. $N=3$. Significance is indicated by $* p<0.05$ compared to control treatment. A. Representative western blot of phosphor AKT, AKT, phosphor p38, p38, phosphor ERK1/2 and ERK1/2 B. Densitometric quantification of phosphor AKT/AKT normalized to control C. Densitometric quantification of phosphor p38/p38 normalized to control D. Densitometric quantification of phosphor ERK1/2/ ERK1/2 normalized to control. Data expressed as mean \pm SEM. $N=3$. Significance is indicated by $* \mathrm{p}<0.05$ compared to control treatment.

proximal tubular cells (Fig. 7B). This indicates blocking CB2 increases albumin endocytosis in proximal tubule cells independently of ERK1/2 signaling pathways. 
Fig. 6. HK2 cells treated with ERK1/2 inhibitor U0126 in conjunction with high albumin (HA; $1 \mathrm{mg} / \mathrm{ml}$ ) and a combination of high glucose/ high albumin (HG; $25 \mathrm{mM} / \mathrm{HA}$ ). Protein abundance (y axis) was normalized to control treatment ( $5 \mathrm{mM}$ glucose, no albumin), data expressed as mean \pm SEM. $N=3$. A. Representative Western blot B. Densitometric quantification of CB2 receptor expression. Significant reductions in CB2 expression was seen in all treatment groups compared to control.

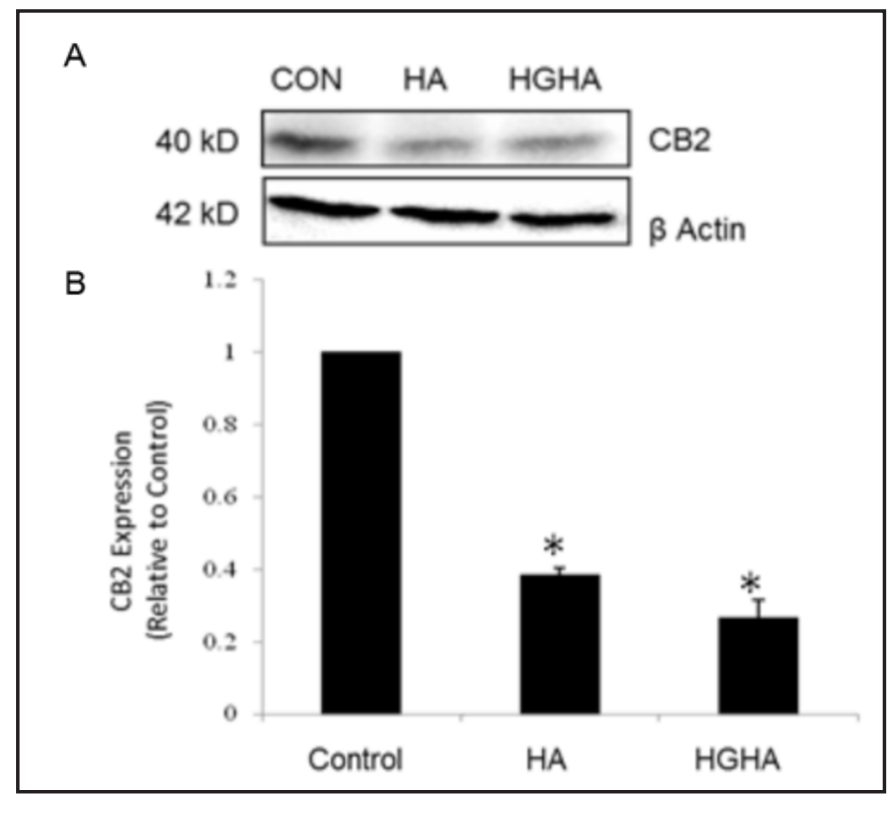

Fig. 7. Albumin uptake in proximal tubule OK cells. Albumin uptake (y axis) was adjusted for background and normalized to control treatment (no inhibitor), data expressed as mean \pm SEM. $N=$ 8. A. OK cells treated with $\mathrm{CB} 2$ inhibitor AM630 had significantly higher albumin uptake compared to control treatment $(\mathrm{p}<0.05)$ B. OK cells treated with ERK1/2 inhibitor U0126 or MAPK inhibitor SB203580 showed no significant difference in albumin uptake compared to control treatment.

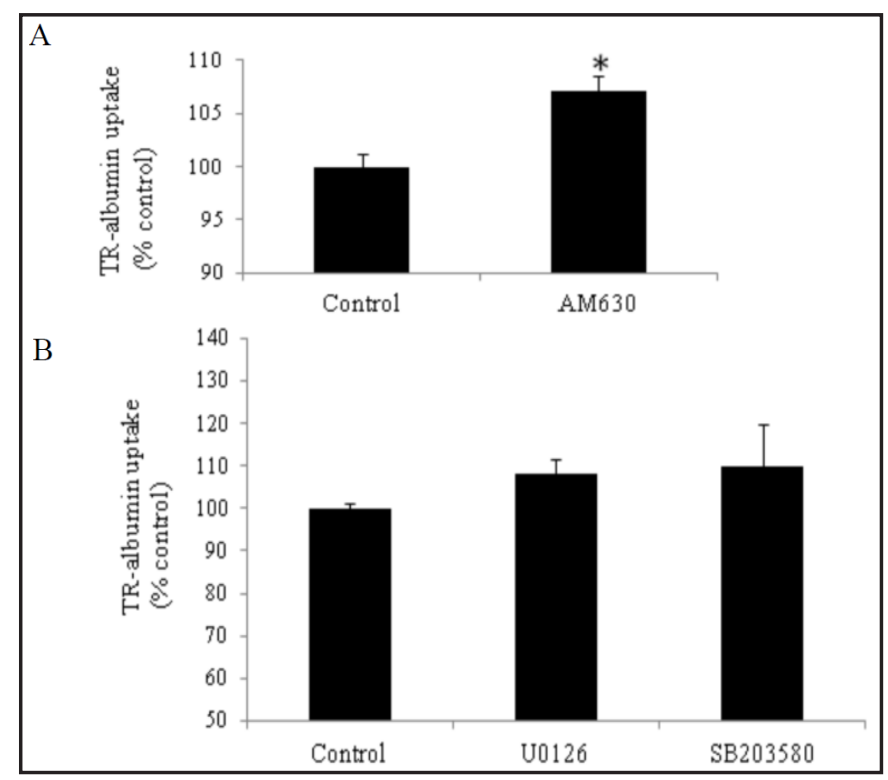

\section{Discussion}

A reduced expression of $\mathrm{CB} 2$ in the diabetic kidney has been targeted in the development of therapeutics for diabetic nephropathy [2]. Down-regulation of CB2 protein in the glomerulus of animals and humans with diabetes may lead to albuminuria, with activation of this receptor reversing this phenotype [2]. Importantly, both glomerular and tubular dysfunction leads to defects in the albumin handling pathway in the kidney [25]. To add to our understanding of the role of CB2 in diabetic nephropathy, our study is the first to show that CB2 expression in proximal tubule cells in vitro can be modulated by high albumin $(1 \mathrm{mg} / \mathrm{ml})$ in the presence and absence of high glucose $(25 \mathrm{mM})$. The reduction in CB2 levels was observed at both the mRNA and protein levels, at specific time points, suggesting sustained exposure to elevated albumin is required to first down regulate CB2 mRNA levels, then consequently CB2 protein. Interestingly, high glucose alone did not significantly alter CB2 receptor mRNA or protein expression at any time point. Potentially, while transient fluctuations in glucose levels does not contribute to alterations in receptor expression at a cellular level in the kidney, it can alter cannabinoid signaling pathways which 
may contribute to the eventual decline in CB2 receptor expression. In vitro, hyperglycemic conditions sustained for 24 hours in pancreatic islet cells, led to changes to calcium signaling but the effect is not mediated by CB2, but by the cannabinoid receptor 1 (CB1) [24]. Further, it has been demonstrated that in early diabetic models of diabetic nephropathy in mice isolated glomeruli showed no significant differences in CB2 expression, while in humans patients with advance diabetic nephropathy have significantly reduced CB2 receptor expression in the renal cortex [2]. We have added to this knowledge, demonstrating that despite a reduction in CB2 expression in specific cells within kidney, in the whole kidney of STZ animals, expression of the CB2 receptor is the same as control animals.

CB2 expression in podocytes [2] and tubular cells is reduced (data presented here). Why then in an in vivo setting, is the expression of CB2 protein in diabetic (STZ) rats unaltered? It is well established that the CB2 receptor is highly expressed in cells of the immune system and assume a diverse range of functions mediating immunological processes in health and disease states $[13,26,27]$. Specifically in diabetic nephropathy, advancement of the renal damage leads to an increase in infiltrating macrophages, T-lymphocytes and the subpopulation of regulatory $\mathrm{T}$ cells which leads to inflammation [28]. In diabetic STZ animals, hallmark indicators of renal damage are elevated such as TGF- $\beta$ and fibronectin, in addition to the development of albuminuria [1]. Significantly, there is also an infiltration of macrophages, which abundantly express CB2 [29]. Thus, it is likely that the CB2 receptor expression in whole kidney samples is influenced by infiltrating macrophages offsetting the downregulation of CB2 in podocytes and proximal tubule cells. In support of this, in the glomeruli from STZ treated animals, there is an infiltration of CB2-expressing macrophages [2].

Our finding presented in this study, that blocking CB2 leads to an increase in albumin uptake, does not directly account for the albuminuria that is observed in animal and human models of diabetes [2]. One must consider that diabetic nephropathy is associated with tubular damage primarily due to the exposure to elevated levels of albumin and glucose [1]. Studies have shown that exposure to elevated albumin specifically, results in proximal tubular cells secreting cytokines such as transforming growth factor- $\beta 1$ (TGF- $\beta 1$ ), endothelin- 1 , monocyte chemoattractant protein-1 (MCP-1), regulated upon activation, normal $\mathrm{T}$ cell expressed and secreted (RANTES), interleukin-8, and fractalkine. These secreted cytokines recruit and stimulate interstitial macrophages, which are likely to transform interstitial cells into myofibroblasts, resulting in inflammation and fibrosis in vivo (for review see [14]). Our research demonstrates that in tubular cells, exposure to elevated albumin in the presence and absence of high glucose alters CB2 expression and blocking the CB2 receptor increases albumin uptake, which in vivo, would activate fibrotic damage. Interestingly, a role for TGF- $\beta 1$ in the modulation of CB2 renal expression levels has been suggested by Barutta et al. [2]. Thus it appears that reduction in CB2 levels is an important component of the fibrotic cascade in response to elevated albumin.

The AKT/MAPK signaling cascade is known to mediate to some degree almost every structural change characteristic of diabetic nephropathy, including hypertrophy, cellular stress pathways and apoptosis [30]. Here we have demonstrated that changes in CB2 receptor expression corresponds to changes in ERK1/2 profile, but not AKT or p38. While High albumin alone and in combination of high glucose significantly reduced CB2 levels in proximal tubule cells, treatment of HK2 cells with ERK1/2 antagonist does not affect CB2 receptor expression, indicating that ERK1/2 does not mediate CB2 expression in proximal tubule cells. However, a link between the activity of the CB2 receptor and ERK1/2 has been established $[18,19]$. In vitro CB2 activation results in reduced phosphorylated ERK1/2 protein expression [19] and conversely, CB2 antagonists can lead to greater and sustained activation of MAPK proteins [18], indicating that the mechanism of the CB2 receptor in diabetic nephropathy may mediate changes via the ERK1/2 proteins, while the AKT/MAPK pathway does not directly mediate changes to CB2 receptor expression.

Diabetic nephropathy is associated with glomerular and proximal tubule damage that leads to albuminuria [6]. A recent study demonstrated that activation of CB2 ameliorated 
diabetes induced albuminuria in vivo [2]. In addition, others have shown that activation of CB2 attenuates renal apoptosis [8], hypertrophy [21] and inflammatory mediators $[2,8,31]$. Collectively, these data suggest that CB2 plays a protective role in renal cell physiology, presumably by abating cellular damage caused by inflammatory mediators $[2$, 8]. Downregulation of CB2 in podocytes in the diabetic model has been described [2], but the specific mechanism for this has not been investigated. Upstream damage to the glomerulus results in proximal tubule cells being exposed to higher levels of albumin, but these cells are also responsible for endocytosis of albumin for its reabsorption. Diabetic nephropathy leads to increase workload of tubular reabsorption of albumin. We have showed that by blocking the CB2 receptor with antagonist AM630 leads to a significant increase in albumin uptake by proximal tubule cells, independent of ERK1/2 signaling. Inhibition of ERK1/2 with antagonists showed no changes to albumin uptake under physiological conditions. Further, here we demonstrate that internalization of albumin by proximal tubule cells, and not increased exposure to albumin alone regulates CB2 levels in proximal tubule cells. This may indicate that a possible cross regulatory loop between albumin endocytosis and CB2 receptor expression occurs in proximal tubule cells. Complex cross regulation pathways of CB2 have been identified in other cell types [18, 32].

In summary, our data demonstrated that CB2 receptor expression in proximal tubule cells is modulated by internalization of albumin. Activity of albumin uptake by proximal tubule cells may be mediated by CB2 independent of ERK1/2 signaling. Alterations in CB2 expressions may lead to changes in ERK1/2 signaling, however this pathway does not mediate changes to albumin handling by tubular cells. In addition to this, our study has demonstrated that CB2 protein expression is unaltered in the whole kidney from diabetic animals, suggesting that infiltration of inflammatory cells is influencing the whole renal expression levels of CB2. Future studies should characterize signaling targets that are altered in response to altered CB2 expression in diabetic models in vivo, to ensure that therapeutic modulation of CB2 is an effective therapy for diabetic nephropathy.

\section{Acknowledgements}

KAJ is supported by an Australian Postgraduate Award (APA) scholarship. AJM is supported by an Australian government collaborative research network (CRN) program. This work was supported by a grant from the Allen Foundation (2011.385).

\section{References}

1 Vallon V: The proximal tubule in the pathophysiology of the diabetic kidney. Am J Physiol- Regul Integr Comp Physiol 2011;300:R1009-R1022.

- Barutta F, Piscitelli F, Pinach S, Bruno G, Gambino R, Rastaldi MP, Salvidio G, Di Marzo V, Cavallo Perin P, Gruden G: Protective Role of Cannabinoid Receptor Type 2 in a Mouse Model of Diabetic Nephropathy. Diabetes 2011;60:2386-2396.

3 Qi W, Chen X, Poronnik P, Pollock CA: Transforming growth factor-b/connective tissue growth factor axis in the kidney. Int J Biochem Cell Biol 2008;40:9-13.

4 Comper WD, Hilliard LM, Nikolic-Paterson DJ, Russo LM: Disease-dependent mechanisms of albuminuria. Am J Physiol-Renal Physiol 2008;295:F1589.

5 Jenkin KA, Verty ANA, McAinch AJ, Hryciw DH: Endocannabinoids and the renal proximal tubule: An emerging role in diabetic nephropathy. Int J Biochem Cell Biol 2012;44:2028-2031.

6 Barutta F, Corbelli A, Mastrocola R, Gambino R, Di Marzo V, Pinach S, Rastaldi M, Perin P, Gruden G: Cannabinoid Receptor 1 Blockade Ameliorates Albuminuria in Experimental Diabetic Nephropathy. Diabetes 2010;59:1046-1054.

7 Mukhopadhyay P, Pan H, Rajesh M, Bátkai S, Patel V, Harvey-White J, Mukhopadhyay B, Haska G, Gao B, Mackie K: CB1 cannabinoid receptors promote oxidative/nitrosative stress, inflammation and cell death in a murine nephropathy model. Br J Pharmacol 2010;160:657-668. 


\section{Cellular Physiology Cell Physiol Biochem 2013;32:1309-1319 \begin{tabular}{l|l|l} 
DOI: $10.1159 / 000354529$ & C 2013 S. Karger AG, Basel
\end{tabular} and Biochemistry

8 Mukhopadhyay P, Rajesh M, Pan H, Patel V, Mukhopadhyay B, Bátkai S, Gao B, Hasko G, Pacher P: Cannabinoid-2 receptor limits inflammation, oxidative/nitrosative stress, and cell death in nephropathy. Free Radic Biol Med 2010;48:457-467.

-9 Muccioli GG: Endocannabinoid biosynthesis and inactivation, from simple to complex.Drug Discov Today 2010;15:474-483.

10 Di Marzo V: The endocannabinoid system in obesity and type 2 diabetes. Diabetologia 2008;51:1356-1367.

11 Janiak P, Poirier B, Bidouard J, Cadrouvele C, Pierre F, Gouraud L, Barbosa I, Dedio J, Maffrand J, Le Fur G: Blockade of cannabinoid CB1 receptors improves renal function, metabolic profile, and increased survival of obese Zucker rats. Kidney Int 2007;72:1345-1357.

12 Lim JC, Lim SK, Han HJ, Park SH: Cannabinoid receptor 1 mediates palmitic acid-induced apoptosis via endoplasmic reticulum stress in human renal proximal tubular cells. J Cell Physiol 2010;225:654-663.

13 Pacher P, Mechoulam R: Is lipid signaling through cannabinoid 2 receptors part of a protective system? Prog Lipid Res 2011;50:193-211.

14 Christensen EI, Verroust PJ: Interstitial fibrosis: tubular hypothesis versus glomerular hypothesis. Kidney Int 2008;74:1233-1236.

15 Rane MJ, Song Y, Jin S, Barati MT, Wu R, Kausar H, Tan Y, Wang Y, Zhou G, Klein JB: Interplay between Akt and p38 MAPK pathways in the regulation of renal tubular cell apoptosis associated with diabetic nephropathy. Am J Physiol-Renal Physiol 2010;298:F49-F61.

-16 Nishimoto S, Nishida E. MAPK signalling: ERK5 versus ERK1/2. EMBO rep 2006;7:782-786.

17 Feliers D, Kasinath BS: Erk in kidney diseases. J Signal Transduct 2011;2011:768512.

18 Correa F, Docagne F, Mestre L, Clemente D, Hernangómez M, Loría F, Guaza C: A role for CB2 receptors in anandamide signalling pathways involved in the regulation of IL-12 and IL-23 in microglial cells. Biochem Pharmacol 2009;77:86-100.

19 Romero-Sandoval EA, Horvath R, Landry RP, DeLeo JA: Cannabinoid receptor type 2 activation induces a microglial anti-inflammatory phenotype and reduces migration via MKP induction and ERK dephosphorylation. Mol Pain 2009;5:25-40.

20 Phillips A, Steadman R: Diabetic nephropathy: the central role of renal proximal tubular cells in tubulointerstitial injury. Histol Histopathol 2002;17:247-252.

21 Jenkin KA, McAinch AJ, Grinfeld E, Hryciw DH: Role for Cannabinoid Receptors in Human Proximal Tubular Hypertrophy. Cell Physiol Biochem 2010;26:879-886.

-22 Zafiriou S, Stanners SR, Polhill TS, Poronnik P, Pollock CA: Pioglitazone increases renal tubular cell albumin uptake but limits proinflammatory and fibrotic responses. Kidney Int 2004;65:1647-1653.

23 Hryciw D, Pollock C, Poronnik P: PKCa mediated remodeling of the actin cytoskeleton is involved in constitutive albumin uptake by proximal tubule cells. Am J Physiol-Renal Physiol 2005;288:F1227.

$\checkmark 24$ Juan-Picó P, Fuentes E, Javier Bermudez-Silva F, Javier Diaz-Molina F, Ripoll C, Rodríguez de Fonseca F, Nadal A: Cannabinoid receptors regulate $\mathrm{Ca}^{2+}$ signals and insulin secretion in pancreatic $\beta$-cell. Cell Calcium 2006;39:155-162.

25 Christensen E, Verroust P, Nielsen R: Receptor-mediated endocytosis in renal proximal tubule. Pflügers Archiv 2009;458:1039-1048.

26 Cabral G, Griffin-Thomas L: Emerging Role of the CB2 Cannabinoid Receptor in Immune Regulation and Therapeutic Prospects. Expert Rev Mol Med 2010;11:e3.

27 Tanasescu R, Constantinescu CS: Cannabinoids and the immune system: An overview. Immunobiol 2010;215:588-597

28 Lim AKH, Tesch GH: Inflammation in Diabetic Nephropathy. Mediators Inflamm 2012:2012:146154.

-29 Horváth B, Mukhopadhyay P, Kechrid M, Patel V, Tanchian G, Wink DA, Gertsch J, Pacher P: $\beta$-Caryophyllene ameliorates cisplatin-induced nephrotoxicity in a cannabinoid 2 receptor-dependent manner. Free Radic Biol Med 2012;52:1325-1331.

30 Tang SCW, Lai KN: The pathogenic role of the renal proximal tubular cell in diabetic nephropathy. Nephrol Dial Transplant 2012;27:3049-3056.

-31 Gardner B, Zu LX, Sharma S, Liu Q, Makriyannis A, Tashkin DP, Dubinett SM: Autocrine and Paracrine Regulation of Lymphocyte CB2 Receptor Expression by TGF- $\beta$. Biochem Biophys Res Comm 2002;290:9196.

-32 Lakshmanan AP, Thandavarayan RA, Watanabe K, Sari FR, Meilei H, Giridharan VV, Sukumaran V, Soetikno V, Arumugam S, Suzuki K: Modulation of at-1r/mapk cascade by an olmesartan treatment attenuates diabetic nephropathy in streptozotocin-induced diabetic mice. Mol Cell Endocrinol 2012;348:104-111. 